\title{
Nucleolin Binds to the Proliferating Cell Nuclear Antigen and Inhibits Nucleotide Excision Repair
}

\author{
Chonglin Yang ${ }^{1}$, Myoung Sook Kim ${ }^{2}$, Devulapalli Chakravarty ${ }^{3}$, Fred E. Indig ${ }^{4}$ and France \\ Carrier
}

Marlene and Stewart Greenebaum Cancer Center and Department of Radiation Oncology, University of Maryland at Baltimore School of Medicine, Baltimore, Maryland

\begin{abstract}
Nucleolin is over-expressed in malignant tumors and is used as a marker for cell proliferation and to reliably predict tumor growth rate. However, it is not known whether nucleolin expression is directly involved in or is a consequence of carcinogenesis. Using GST-pull down assays, we have determined that the recombinant nucleolin interacts with the Proliferating Cell Nuclear Antigen (PCNA). Coimmunoprecipitation assays indicate that the nucleolin-PCNA interaction also occurs in intact cells and this interaction increases after exposure of colon carcinoma RKO cells to UV radiation. Moreover, our data indicate that PCNA and nucleolin co-localize in some areas within the RKO cell nuclei. The functional significance of this interaction is evaluated on Nucleotide Excision Repair (NER) since PCNA is a primary mediator of this cellular function. Our data indicate that overexpression of nucleolin decreases the repair efficiency of UV damaged plasmid DNA in RKO cells. Co-transfection with PCNA can rescue this effect in vivo. Furthermore, reduction of nucleolin protein levels increases DNA repair efficiency in RKO and CHO cells and consequently increases cell survival. These data indicate that the direct interaction of nucleolin with PCNA inhibits NER efficiency of UV damaged DNA. This effect could contribute to carcinogenesis and aging in cells over-expressing nucleolin.
\end{abstract}

\footnotetext{
Received 06/01/09; accepted 07/20/09

Current addresses: ${ }^{1}$ Institute of Genetics and Developmental Biology, Chinese Academy of Sciences, Datun Road, Chao Yang District, Beijing 100101, China. 2Johns Hopkins University, Baltimore, Maryland. ${ }^{3}$ Visakhapatnam, India. ${ }^{4}$ National Institute on Aging, National Institutes of Health, Baltimore, MD.

Correspondence: France Carrier, Ph.D., Department of Radiation Oncology, University of Maryland at Baltimore School of Medicine, 655 West Baltimore St., Room 10-037, Baltimore, MD 21201, USA. Tel. 410-706-5105, Fax. 410706-3260. email: fcarr001@umaryland.edu
}

Keywords: PCNA; Nucleotide Excision Repair; Genotoxic stress; UV radiation

\section{Introduction}

Nucleolin was initially described as a nucleolar protein participating in ribosome biogenesis (reviewed in (1). Subsequent studies have shown that nucleolin is a multifunctional protein involved in several cellular processes such as transcription (2), attachment of genomic DNA to nuclear matrix and decondensation of chromatin (3). Nucleolin is apparently not restricted to the nucleolus, in some cells nucleolin can localize to the nucleus, the cytoplasm and even the cell surface (4), (5). In addition, specific interactions between nucleolin and a number of nuclear proteins such as nucleophosmin (6), topoisomerase 1 (7) and the growth factor midkine (8) have been reported. Nucleolin is overexpressed in malignant tumors and its synthesis is associated with increased rates of cell division (9). Nucleolin is one of the major components of the silver-stained nucleolar organizer regions (AgNORs) that are used as markers for cancer progression in several tumor types (9). However, the precise mechanism(s) by which nucleolin could contribute to cancer progression are still poorly understood.

A possible role for nucleolin in recombinational DNA repair has been suggested due to its ability to accelerate the annealing of oligonucleotides, including those containing mismatches (10). In addition, nucleolin helicase activity and its capacity to bind to hairpin RNA structures (11), may also indicate a possible role in other DNA repair mechanisms such as transcription-coupled repair or nucleotide excision repair (NER). PCNA is overexpressed during the S-phase of the cell cycle and is used as a marker of cell proliferation (12). PCNA functions in DNA replication as a molecular sliding clamp that permits highly processive synthesis of 
DNA polymerase $\delta$ (13). The sliding and scaffolding properties of PCNA are also important for DNA repair, including NER. In fact, decreased levels of PCNA mRNA and protein levels are associated with reduced repair capacity of UV damaged DNA in aged human fibroblasts (14). PCNA interacts with several proteins that are involved in either DNA replication, repair or cell-cycle regulation (12). The mechanisms that modulate the interaction of PCNA with distinct binding partners are not fully understood but evidences indicate that posttranslational modifications are important (15). It thus appears that these interactions are not random but functionally regulated. Here we demonstrate that PCNA interacts with Nucleolin and is functionally affected by Nucleolin levels in the cells.

\section{Material and Methods \\ Plasmids, Cell lines and treatments}

To generate the sense and antisense Nucleolin vectors, the Open Reading Frame (ORF) of the human Nucleolin was cloned in the sense and antisense orientation into the $\mathrm{KPnI} / \mathrm{XhoI}$ sites of pcDNA3.1 (Invitrogen, Carlsbad, CA). The human colorectal carcinoma RKO cells were maintained in RPMI 1640 medium (Gibco-BRL, Rockville, MD) supplemented with $10 \%$ fetal bovine serum. The nucleolin antisense cell line was established by stable transfection of the nucleolin expression vector containing the ORF in the antisense orientation. Single colonies were expanded and expression levels of Nucleolin were verified by Western blot. Clonogenic survival after UV irradiation was performed by colony formation assay. About 200 cells were seeded per $100 \mathrm{~mm}$ dish for overnight growth, then the medium was removed and the cells were irradiated with the indicated dose of UV. Treatments were performed in triplicate for each dose. Two weeks later, colonies containing more than 50 cells were counted. UV treatment was performed as described previously (16) except that the source of UVC was a Philips $30 \mathrm{~W}$ germicidal lamp emitting at $253.7 \mathrm{~nm}$ and the intensity of the UV lamp was determined with a UVX Radiometer (UVP Inc., Upland, CA).

\section{MTT assay}

The assay was performed as described before (17). Briefly, the cells were plated at $1 \times 10^{4}$ per 24 wells plate, $72 \mathrm{hr}$ after UV exposure $(7 \mathrm{Jm}-2)$ the cells were incubated with the tetrazolium salt, MTT $(0.25 \mathrm{mg} / \mathrm{ml}$ in PBS) for $3 \mathrm{hr}$. The media was then removed, DMSO (1 $\mathrm{ml}$ of $100 \%$ ) was added and viability was determined by absorbance at $540 \mathrm{~nm}$. Each sample was assayed at least three times. The results were expressed as a percentage of MTT reduction in treated samples compare to untreated sample (100\%).

\section{Co-immunoprecipitation and Western blot}

The DNA fragment encoding nucleolin residues 284 to 707 (Nuc-C) was amplified by PCR and cloned into the BamHI/XhoI sites of the bacteria expression vector pGEX-4T (Amersham). The protein was expressed in BL21(DE3) with 0.4mM IPTG. To pull down recombinant PCNA, the reaction mixture $(100 \mu \mathrm{l})$ contained $30 \mu \mathrm{l} 50 \%$ slurry of glutathione Sepharose 4B (Amersham), 10 $\mu$ l PCNA-containing bacterial supernatant and 500ng GST protein or GST-Nuc-C having an equimolar ratio to GST. The reactions were performed at $4^{\circ} \mathrm{C}$ and the glutathione beads were washed 5 times with RIPA ( $(50 \mathrm{mM}$ Tris$\mathrm{HCl}, \mathrm{pH} 7.5 ; 150 \mathrm{mM} \mathrm{NaCl}, 1 \% \mathrm{NP}-40,0.5 \%$ sodium deoxycholate, $0.1 \%$ SDS) buffer. The bound proteins were separated on $12 \%$ SDS-PAGE. Western blots were performed with PCNA antibody (Oncogene, San Diego, CA). The purified human GST-PCNA (1 $\mu \mathrm{g})$ was obtained from Proteomix (Baltimore, MD) and incubated with purified recombinant Nuc-C (1 $\mu \mathrm{g})$. The bound proteins were eluted from glutathione sepharose as described above and Western blot for nucleolin. For immunoprecipitation in intact cells, the human colorectal carcinoma cells (RKO, (16)) were grown and irradiated (UVC, $14 \mathrm{Jm}^{-}$ 2) as described before (16). The cells were lysed in RIPA buffer and used for co-immunopreciopitation or Western Blot. Total cell extracts $(300 \mu \mathrm{g})$ were immunoprecipitated with PCNA antibody and Western blots were performed with nucleolin (18) and PCNA antibodies (1:2000 dilution).

\section{Immunofluorescence}

The RKO cells were cultured on cover slips. After overnight growth, the cells were fixed and immunofluorescence microscopy was performed with nucleolin polyclonal antibody (1:500 dilution) stained with rodamine-conjugated goat anti-rabbit IgG (1:50 dilution) and PCNA monoclonal antibody (1:500 dilution) stained with fluorescin-conjugated anti-mouse IgG (1:50 dilution). Nuclear content was visualized by DAPI staining. The cover slips were mounted and observed under a fluorescence microscope (TE200 Nikon). 


\section{Nuclear Excision Repair efficiency in a host cell re-activation assay}

The RKO cells were transiently transfected with $1 \mu \mathrm{g}$ of empty vectors or pCMV.3-Nucleolin plasmid and/or pcDNA3.1-PCNA plasmid using LipofectaminePlus ${ }^{\mathrm{TM}}$ reagents (Invitrogen). The total amounts of transfected DNA were kept constant with empty plasmid vectors. Twenty four hours later, the cells were co-transfected with $50 \mathrm{ng}$ of either sham or UVC light exposed $\left(0.5 \mathrm{KJm}^{-2}\right)$ Luciferase reporter plasmid pGL-3 plasmid (0.1 $\mathrm{mg} / \mathrm{ml}$ in double distilled $\mathrm{H}_{2} \mathrm{O}$ ) and $10 \mathrm{ng}$ of $\mathrm{pRL}$ SV40 plasmid for the transfection control. The Luciferase assay was performed 24 hours later according to the manufacturer (Promega) recommendation. The pGL-3 luciferase activity was determined by a luminometer (Model; Optocomp I Luminometer, MGM Instruments Inc., Hamden, CT). The pRL luciferase activity was subsequently determined by adding $50 \mu \mathrm{l}$ of Stop and Glo $1 \mathrm{X}$ Reagent to the reaction. The relative repair efficiency was calculated by first calculating the relative luciferase activity (RLU) in each sample (pGL-3 luciferase activity/pRL luciferase activity). The repair efficiency (RE) was then calculated as (UV-damaged RLU/undamaged RLU) X 100. The RE of the $0 \mathrm{KJm}^{-2}$ samples was calculated as (undamaged RLU/undamaged RLU) X 100. The relative repair efficiency (RRE) was finally calculated as (R.E. of transfected plasmid/R.E. of empty vector) X 100. For cells transfected with siRNA nucleolin, the percentage of repair efficiency was calculated with results obtained with a control siRNA.

\section{siRNA}

The nucleolin small interfering RNA (SiRNA) duplexes were synthesized by Qiagen-Xeragon (Germantown, MD) and transfected into RKO or CHO-K1 cells using oligofectamine (Invitrogen) as recommended by the manufacturer. The hamster CHO-K1 cells were from American Type Culture Collection (Manassas, VA) and grown as the RKO cells. Forty-eight hours after transfection, the cells were co-transfected with pGL-3 and pRL as described above. The SiRNA sequence-targeting nucleolin corresponded to nucleotides 626-646 of the coding region relative to the first nucleotide of the start codon (sense; 5'-GCUAUGGAGACUACACC AG-3'; antisense; 5'-CUGGUGUAGUCUCCAUAGC3 '). The negative control was SiRNA for S100ß. The 23 nucleotides (nt) double stranded RNA sequence $(5>$ ACU ACU GCC UGC CAC GAG UUC<3') corresponded to S100 $\beta$ C-terminal end (nt 244-265) plus two dT 3 ' overhang.

\section{Results and Discussion}

Because nucleolin and PCNA are both associated with cell proliferation and are used as markers for cancer progression (9), we wanted to determine whether nucleolin could interact with PCNA. We first performed co-immunoprecipitation with recombinant proteins. The NH2 terminal domain of nucleolin is very acidic and often hinders the solubility of the recombinant protein in addition to causing anomalous migration of the protein on SDSPAGE (19). Therefore, we deleted the first 283 amino acids of the protein and generated a recombinant protein containing the four RNA binding domains (RBD) and the C-terminal arginine-glycine rich domain (RGG box) (Nuc-C) of nucleolin (Figure 1A). The GST-fusion protein was incubated with bacterial extracts over-expressing PCNA and eluted on Glutathione Sepharose 4B by standard procedure. Our data (Figure 1B) indicate that the nucleolin deletion mutant interacts with PCNA and suggest that the N-terminal end of nucleolin is not required for this interaction. Furthermore, we also verified the interaction by using GST-PCNA and purified recombinant Nuc-C. Our data (Figure 1C) indicate that PCNA also binds to Nuc-C. The additional bands that are also detected by the nucleolin antibody are likely the result of the intrinsic self cleaving activity of nucleolin. In fact, this self-cleaving activity, also known as maturation, has been observed in the full length Nucleolin as well as in a two-third deletion mutant similar to the one used here(20,21). This self cleaving activity is cell-type-specific and sensitive to protease inhibitors (20). It thus seems possible that other self cleaved NCL fragments also interact with PCNA.

A number of proteins that interact with PCNA share a common motif called the PCNA interaction protein box (PIP-box). The PIP-box consists of the sequence $\mathrm{Q}-\mathrm{x}-\mathrm{x}-(\mathrm{I} / \mathrm{L} / \mathrm{M})-\mathrm{x}-\mathrm{x}-(\mathrm{F} / \mathrm{Y})-(\mathrm{F} / \mathrm{Y})$ where $\mathrm{x}$ is any residue (22). However, not all PCNA-interacting proteins contain this sequence. For example, cyclin $\mathrm{D}$ and DNA polymerase $\varepsilon$ do not contain the PIP box (13). Another motif called the KA-box has also been identified as a PCNA interacting domain. This conserved sequence is found in DNA polymerase $\delta$ and several other proteins involved in DNA replication, repair and cell cycle regulation (13). Interestingly, we have not found any PIP box in 
A

Nucleolin

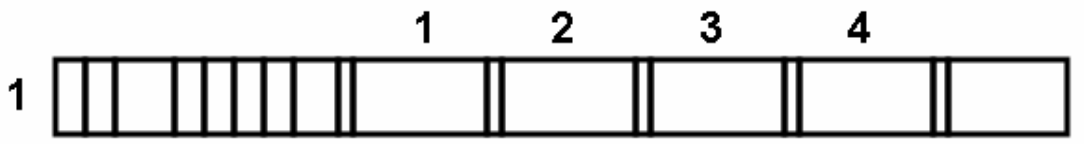

707

Nuc-C

284

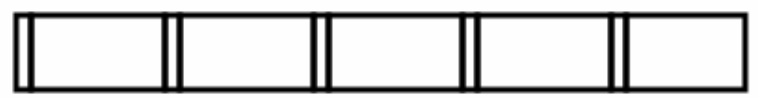

707

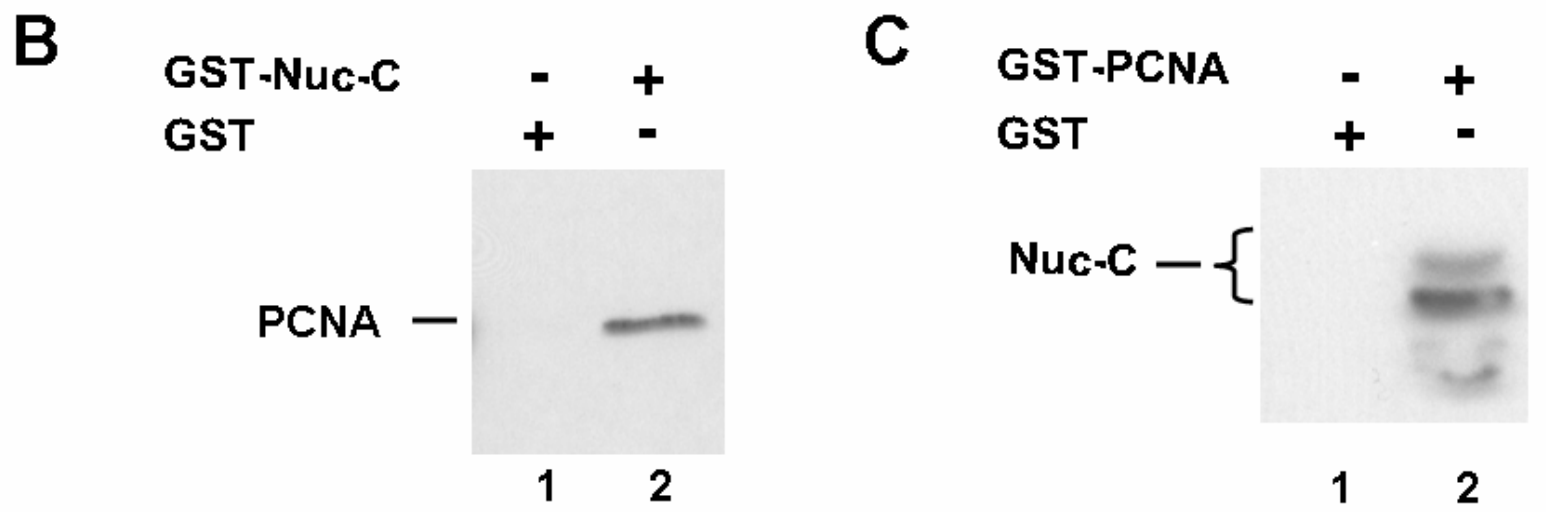

Figure 1. Recombinant Nucleolin binds to PCNA. (A) Schematic representation of the full length nucleolin and the fragment encoding nucleolin residues 284 to 707 (Nuc-C). (B) Nucleolin interacts with PCNA. Recombinant PCNA was incubated with either GST or GST-Nuc-C as described in Material and Methods. The bound proteins were separated on 12\% SDS-PAGE and Western blots with PCNA antibody. (C) The purified human GST-PCNA was incubated with purified recombinant Nuc-C and the eluted proteins were Western blot with nucleolin antobody.

nucleolin but there is a sequence very similar to the KA-box in the RNA Binding Domain 3 (residues 467 to 520) of nucleolin. Whether this sequence is responsible for the interaction remains to be determined.

We then proceeded to determine whether nucleolin could interact with PCNA in vivo. Because nucleolin is a stress-responsive protein and that UV radiation induced binding of $\mathrm{p} 21$ and ING1 to PCNA (23), we also wanted to evaluate the possible effect of UV radiation on the nucleolin-PCNA interaction. We thus performed co-immunoprecipitation with PCNA antibody in colon carcinoma RKO cells at different times following exposure to UV radiation (Figure 2A). Our data indicate that the PCNA antibody precipitated PCNA as well as some nucleolin protein immediately following exposure to UV light (Figure 2A, No Delay). However, the amounts of endogenous nucleolin that co-immunoprecipitated with the
PCNA antibody increased as soon as 15 min after exposure to UV radiation (lane 2) and remained elevated for up to $45 \mathrm{~min}$ (lanes 2-4). The increased amounts of nucleolin in the immunoprecipitate were not due to increased levels of PCNA in the reaction complex since the levels of PCNA were relatively the same in all the samples (Figure 2A, lanes 1-4). To determine whether the overall levels of nucleolin or PCNA were also affected by UV radiation, we performed Western blot analyses on the same extracts before immunoprecipitation. Our data (Figure 2B) indicate that the levels of nucleolin and PCNA did not change in response to UV radiation. What is changed in response to UV radiation is the amount of nucleolin that can be recruited by PCNA. This may suggest that some post-translational modifications activated by UV are mediating the increased interaction. In that regard we have previously shown that UV-induced nucleolin 
A

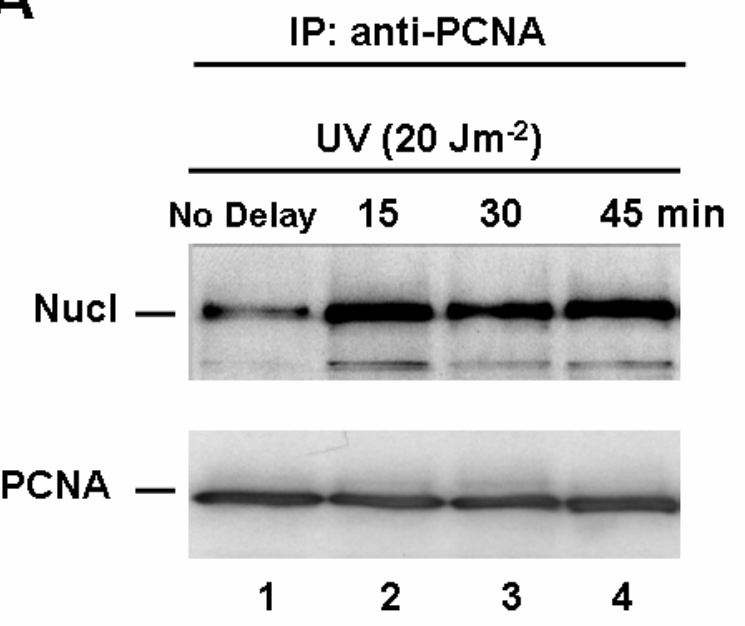

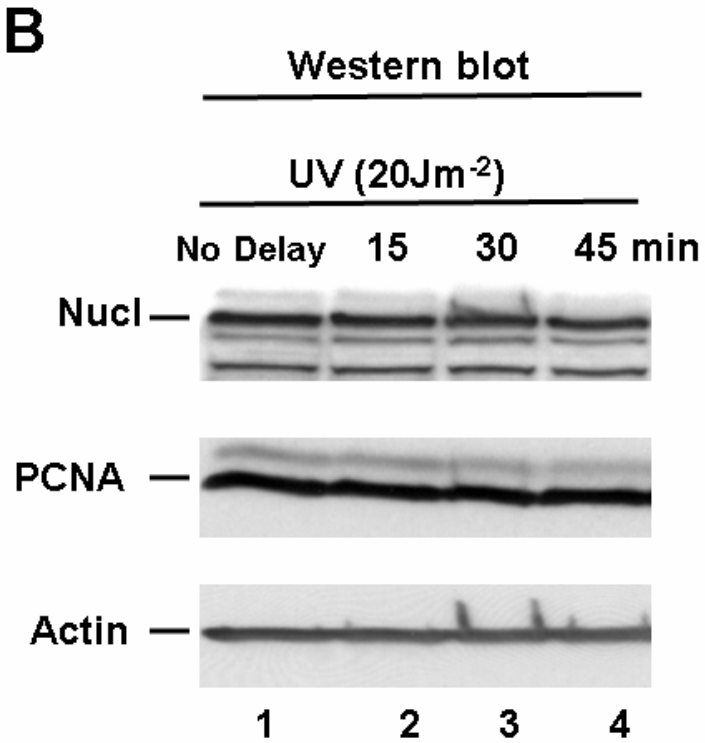

Figure 2. Nucleolin and PCNA interact in intact cells. The RKO cells were grown and irradiated (UVC, $\left.20 \mathrm{Jm}^{-2}\right)$ as described before (16). The cells were lysed and used for co-immunopreciopitation (A) or Western Blot (B) with the indicated antibodies. Nucl (nucleolin), PCNA (Prolefaring Cell Nuclear Antigen). Actin antibody was used to control for equal loading.

phosphorylation increases its RNA binding activity (18). It thus seems possible that UV-induced posttranslational modifications could also influence the nucleolin-PCNA protein interactions. The precise molecular mechanisms that could be responsible for this increased interaction are currently under study.

For an interaction to be functional in vivo the proteins have to be localized in the same cellular compartment. We used immunofluorescence to determine whether nucleolin and PCNA are colocalized in vivo. Asynchronous RKO cells were fixed and stained with a polyclonal antibody for nucleolin and reacted with a goat anti-rabbit secondary antibody conjugated to rhodamine. The cells were also stained with a monoclonal antibody for PCNA and reacted with a fluorescein-conjugated mouse secondary antibody. Our data (Figure 3) indicate that both PCNA and nucleolin are found in the nucleus and the nucleolus and that their respective staining overlay in several areas suggesting that the interaction could have a functional significance in vivo.

Because UV radiation increased the interaction of nucleolin with PCNA (Figure 2) and since PCNA is involved in the repair of UV damaged DNA (22), we wanted to determine whether nucleolin could affect PCNA repair activity on UV damaged DNA. We used the plasmid host cell re-activation assay to measure DNA repair efficiency. A UVC-irradiated

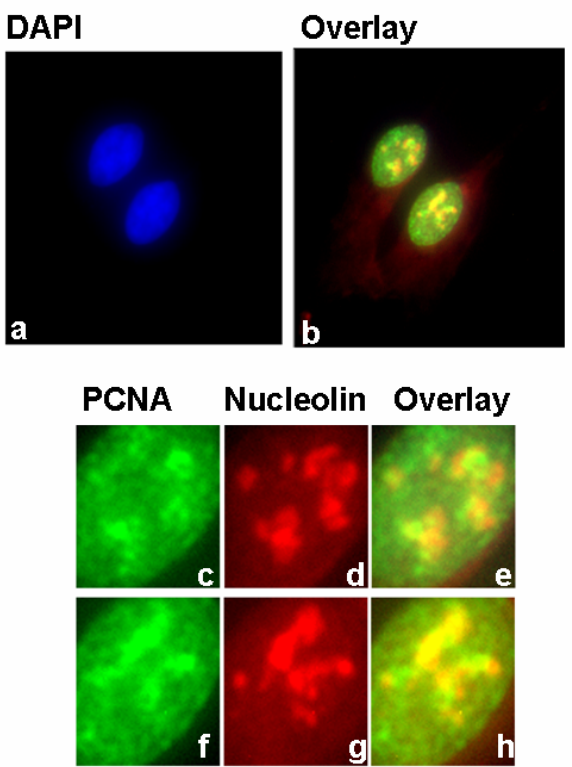

Figure 3. Nucleolin and PCNA colocalize in some areas of RKO cells. The RKO cells were cultured on cover slips, fixed and photographed under immunofluorescent microscope (TE200 Nikon). (a) DAPI staining, (c) and (f) PCNA (fluorescin-conjugated) top and bottom cell respectively from panel (a) enlarged 3 times $(3 X)$, (d) and (g) Nucleolin (rodamine-conjugated) top and bottom cell as in (c) and $(\mathrm{f})$, (b) overlay of red and green at lower magnification and $(\mathrm{e})$ and $(\mathrm{h})$ top and bottom cell respectively of panel (b) enlarged $(3 \mathrm{X})$ to better view areas of co-localization. Co-localization is indicated by overlay of the red and green (yellow) staining. 
A

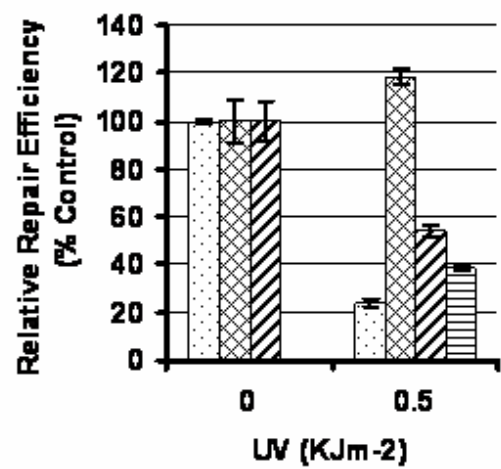

B

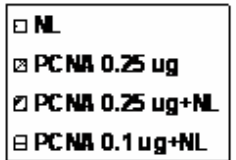

aRKO-siRNA-M

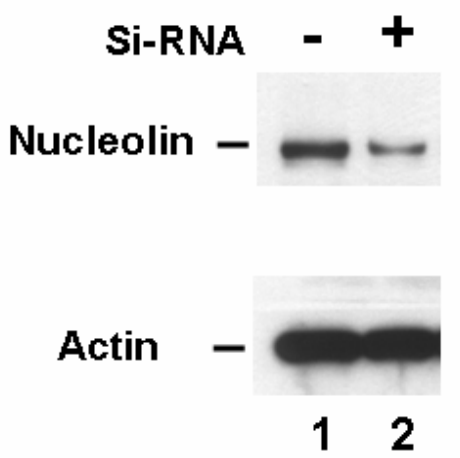

- CHO-SIRNA-NL

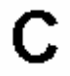

$$
\text { 을 }
$$

250

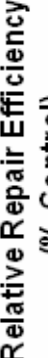

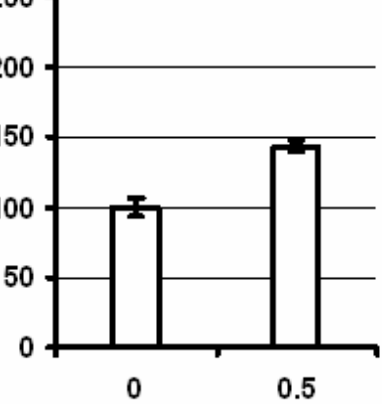

UV (KJm-2)
D

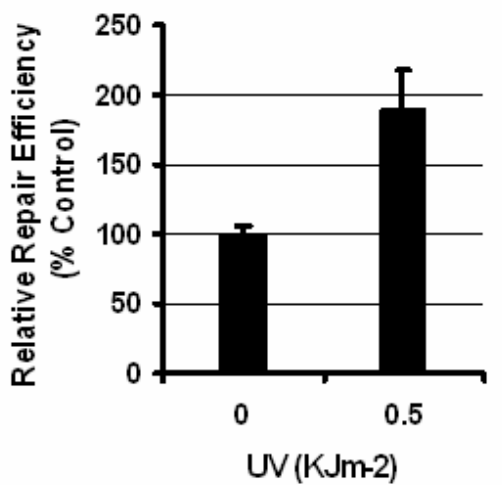

Figure 4. Nucleolin inhibits NER efficiency in a host cell re-activation assay. (A) The RKO cells were transiently transfected with empty vectors or pCMV.3-Nucleolin plasmid and/or pcDNA3.1-PCNA plasmid. Twenty four hours later, the cells were co-transfected with either sham or UVC damaged luciferase reporter plasmid pGL-3 plasmid and pRL-SV40 plasmid for the transfection control. The relative repair efficiency (RRE) was calculated as described under Material and Methods. (B) The RKO cells were transfected with nucleolin siRNA and the levels of nucleolin were measured by Western blot. Actin was used as a control for loading. (C) Host cell reactivation was performed as in (A) except that the RKO cells were transfected with nucleolin siRNA. The percentage control efficiency was calculated with results obtained with a control siRNA. (D) Host cells reactivation as in (C) except that the experiments were conducted in CHO cells.

luciferase reporter construct (pGL-3) was used to measure the DNA-repair ability in different RKO cells transiently transfected with either empty vector, nucleolin, PCNA or nucleolin and PCNA (Figure 4). This assay reflects the NER of damaged DNA (for TT or TC dimers) by using transcriptional activity measured as luciferase enzyme activity of the transfected reporter gene. The pGL-3 plasmid was either sham irradiated $\left(0 \mathrm{KJm}^{-2}\right)$ or exposed to UVC light $(254 \mathrm{~nm})$ at $0.5 \mathrm{KJm}^{-2}$. Because the relative repair efficiency is expressed as a percentage of the relative luciferase activity (pGL$3 / \mathrm{pRL}$ ) in each sample, the relative repair efficiency in the absence of $\mathrm{UV}$ radiation $\left(0 \mathrm{KJm}^{-2}\right)$ is about
$100 \%$ in all samples (Figure 4A). However, the relative repair efficiency of cells transiently transfected with nucleolin alone decreased by almost $80 \%$. On the other hand, transfecting the cells with PCNA alone did not affect the repair capacity of the cells and even slightly increased it. In addition, when the cells were co-transfected with both, PCNA and nucleolin, PCNA was able to rescue the inhibitory effect of nucleolin by more than 50\%. A similar effect was also observed in $\mathrm{CHO}$ cells (data not shown). These data indicate that an in vivo interaction of nucleolin with PCNA can inhibit PCNA function in NER. 
If nucleolin were directly responsible for this effect, reduced levels of nucleolin should result in increased relative repair efficiency. To verify this hypothesis, we decreased the levels of nucleolin by the small interference RNA technique (siRNA) and measured the basal levels of nucleolin in the absence and presence of the nucleolin siRNA by immunoblot analysis (Figure 4B). Under these conditions the basal levels of nucleolin are substantially reduced. In order to ensure that the effect measured would not be an artifact of the transfection technique or of the presence of synthetic RNA, we also performed the experiments with the same concentration of a control siRNA. The relative repair efficiency was then measured and the results were expressed as a percentage of this negative control. As evident from Figure 4C reduced levels of nucleolin in RKO cells result in increased DNA repair efficiency by more than $40 \%$. This effect is even more pronounced in CHO cells where reduced levels of nucleolin almost double (190\%) the NER efficiency (Figure 4D). These data indicate that nucleolin is directly involved in the regulation of NER efficiency and can affect more than one cell type. The pronounced effect in $\mathrm{CHO}$ cells may be due to the fact that nucleolin is more responsive to UV radiation in these cells (24). In fact, we have previously shown that UV-induced nucleolin phosphorylation increased its RNA binding activity by several folds in these cells as compared to other cell lines $(18,24)$. We can not thus rule out the possibility that the levels of UV-induced nucleolin post-translational modifications are different in CHO cells and affect nucleolin protein-protein interaction as well as its role in NER efficiency.

Increasing the capacity of a cell to repair its damaged DNA should result in increased survival. To verify that the increased NER efficiency observed in the presence of reduced levels of nucleolin is actually affecting cell survival, we measure cell viability by MTT and colony formation assays under these conditions. The data shown in Figure 5 indicate that indeed when the nucleolin levels are reduced the cells viability is increased by almost two fold (Figure5B) and cell survival is increased by more than 5 fold. This indicates that the effect of nucleolin on NER has direct consequence on the cellular capacity to respond to cellular insults.

The interaction between nucleolin and PCNA is both functional (Figure 4-5) and regulated by UV radiation (Figure 2). Because nucleolin shuttles between the different cellular compartments (4) it is possible that either PCNA or other proteins interacting with PCNA and nucleolin such as the
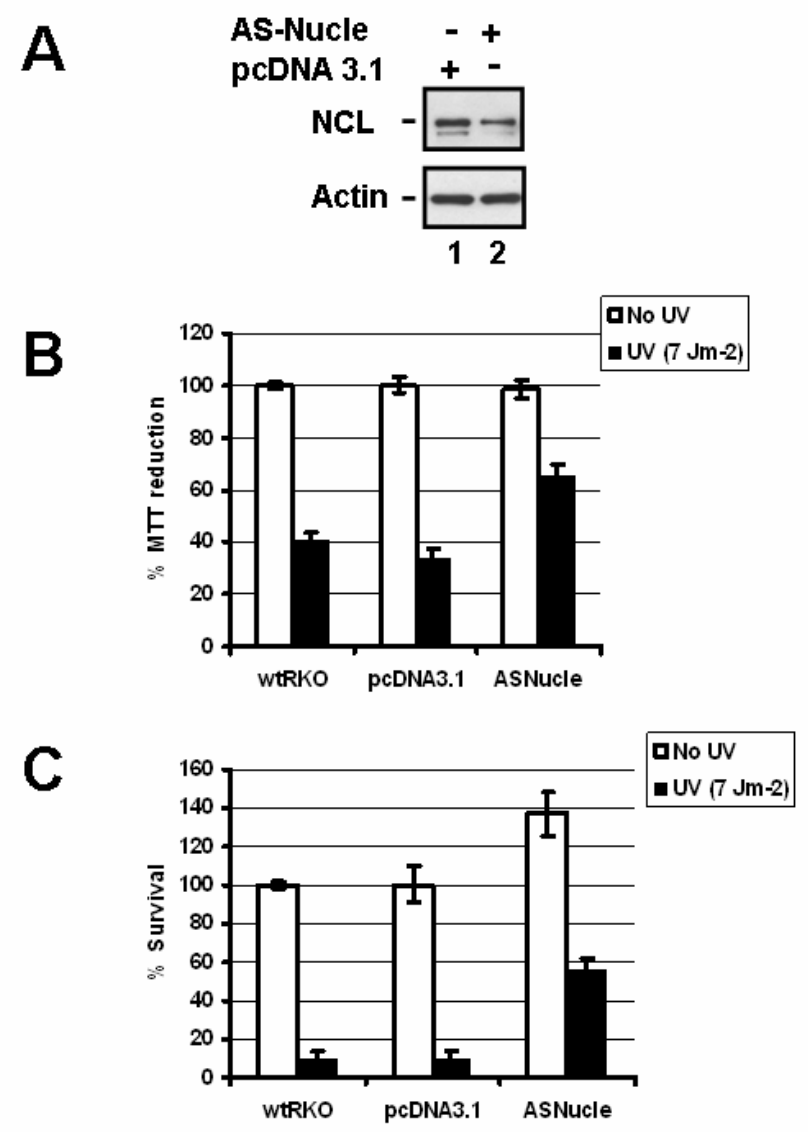

Figure 5. Down regulation of Nucleolin increases cell survival. (A) Western blot of protein extracts obtained from cell transfected with either empty vector (lane 1) or antisense-Nucleolin vector (ASNucle) (lane 2). (B) Cell viability was measured by MTT assay on non-transfected cells (wt RKO), cells transfected with empty vector (pcDNA3.1) or cells transfected with antisense-Nucleolin (ASNucle) vector. Cells were either sham irradiated (No UV) or exposed to 7 Jm-2 UV radiation. MTT assay was performed 72 hours after UV exposure and results are expressed as a percentage of MTT reduction of treated samples compared to untreated samples. (C) Clonogenic survival was performed by colony formation assay on cells transfected as in (B) after $7 \mathrm{Jm}-2$ of UV radiation. Results are expressed as a percentage of surviving colonies over untreated samples.

Replication Protein A (25), (26) could recruit nucleolin at sites of DNA damage in response to UV radiation. This possibility remains, however, to be determined.

Interestingly, a direct correlation has been found between the AgNORs counts, which are mainly composed of nucleolin, and the malignant progression of melanoma (27). The UV-induced binding of nucleolin to PCNA may contribute to this progression. The interaction of nucleolin with PCNA could also have consequences in aging. Older cells, including aged human fibroblasts, have a reduced rate of removal of thymidine dimers and (6-4) 
photoproducts (14). This has been attributed to a decrease of expression of several proteins involved in NER including PCNA. The interaction of nucleolin with PCNA could thus also participate in the aging process by decreasing NER efficiency.

In summary, our data indicate that nucleolin interacts directly with PCNA and that this interaction is increased by UV radiation in vivo. Nucleolin interaction with PCNA reduces NER efficiency and consequently affects cell survival. The over-expression on nucleolin could thus contribute to carcinogenesis and aging.

\section{Acknowledgements}

This work was supported in part by a grant from the National Institutes of Health 1RO1GM57827-01 (FC).

\section{Conflicts of Interest}

No potential conflicts of interest to disclose.

\section{References}

1. Srivastava M, Pollard HB. Molecular dissection of nucleolin's role in growth and cell proliferation: new insights. FASEB J 1999;13:1911-22.

2. Ying GG, Proost P, van Damme J, Bruschi M, Introna M, Golay J. Nucleolin, a novel partner for the Myb transcription factor family that regulates their activity. J Biol Chem 2000;275:4152-8.

3. Erard M, Lakhdar-Ghazal F, Amalric F. Repeat peptide motifs which contain beta-turns and modulate DNA condensation in chromatin. Eur J Biochem 1990;191:19-26. 4. Borer RA, Lehner CF, Eppenberger HM, Nigg EA. Major nucleolar proteins shuttle between nucleus and cytoplasm. Cell 1989;56:379-90.

5. Hovanessian AG, Puvion-Dutilleul F, Nisole S, et al. The cell-surface-expressed nucleolin is associated with the actin cytoskeleton. Exp Cell Res 2000;261:312-28.

6. Li YP, Busch RK, Valdez BC, Busch H. C23 interacts with B23, a putative nucleolar-localization-signal- binding protein. Eur J Biochem 1996;237:153-8.

7. Bharti AK, Olson MO, Kufe DW, Rubin EH. Identification of a nucleolin binding site in human topoisomerase I. J Biol Chem 1996;271:1993-7.

8. Take M, Tsutsui J, Obama H, et al. Identification of nucleolin as a binding protein for midkine (MK) and heparin-binding growth associated molecule (HB-GAM). J Biochem (Tokyo) 1994;116:1063-8.

9. Derenzini M, Sirri V, Trere D, Ochs RL. The quantity of nucleolar proteins nucleolin and protein B23 is related to cell doubling time in human cancer cells. Lab Invest 1995;73(4):497-502.

10. Hanakahi LA, Bu Z, Maizels N. The C-terminal domain of nucleolin accelerates nucleic acid annealing. Biochemistry 2000;39:15493-9.
11. Ghisolfi-Nieto L, Joseph G, Puvion-Dutilleul F, Amalric F, Bouvet P. Nucleolin is a sequence-specific RNA-binding protein: characterization of targets on preribosomal RNA. J Mol Biol 1996;260:34-53.

12. Warbrick E. The puzzle of PCNA's many partners. Bioessays 2000;22:997-1006.

13. Xu H, Zhang P, Liu L, Lee MY. A novel PCNA-binding motif identified by the panning of a random peptide display library. Biochemistry 2001;40:4512-20.

14. Goukassian D, Gad F, Yaar M, Eller MS, Nehal US, Gilchrest BA. Mechanisms and implications of the ageassociated decrease in DNA repair capacity. FASEB $J$ 2000;14:1325-34.

15. Matunis MJ. On the road to repair: PCNA encounters SUMO and ubiquitin modifications. Mol Cell 2002;10:4412.

16. Yang C, Carrier F. The UV-inducible RNA-binding protein A18 (A18 hnRNP) plays a protective role in the genotoxic stress response. J Biol Chem 2001;276:47277-84. 17. Kim MS, Blake M, Baek JH, Kohlhagen G, Pommier Y, Carrier F. Inhibition of histone deacetylase increases cytotoxicity to anticancer drugs targeting DNA. Cancer Res 2003;63:7291-300.

18. Yang C, Maiguel DA, Carrier F. Identification of nucleolin and nucleophosmin as genotoxic stressresponsive RNA-binding proteins. Nucleic Acids Res 2002;30:2251-60.

19. Ginisty H, Sicard H, Roger B, Bouvet P. Structure and functions of nucleolin. J Cell Sci 1999;112:761-72.

20. Chen CM, Chiang SY, Yeh NH. Increased stability of nucleolin in proliferating cells by inhibition of its selfcleaving activity. J Biol Chem 1991;266:7754-8.

21. Fang $\mathrm{SH}$, Yeh $\mathrm{NH}$. The self-cleaving activity of nucleolin determines its molecular dynamics in relation to cell proliferation. Exp Cell Res 1993;208:48-53.

22. Paunesku T, Mittal S, Protic M, et al. Proliferating cell nuclear antigen (PCNA): ringmaster of the genome. Int $\mathrm{J}$ Radiat Biol 2001;77:1007-21.

23. Li R, Waga S, Hannon GJ, Beach D, Stillman B. Differential effects by the p21 CDK inhibitor on PCNAdependent DNA replication and repair. Nature 1994;371:534-7.

24. Carrier F, Gatignol A, Hollander MC, Jeang KT, Fornace AJ, Jr. Induction of RNA-binding proteins in mammalian cells by DNA-damaging agents. Proc Natl Acad Sci U S A 1994;91(4):1554-8.

25. Loor G, Zhang SJ, Zhang P, Toomey NL, Lee MY. Identification of DNA replication and cell cycle proteins that interact with PCNA. Nucleic Acids Res 1997;25:50416.

26. Daniely Y, Borowiec JA. Formation of a complex between nucleolin and replication protein A after cell stress prevents initiation of DNA replication. J Cell Biol 2000;149:799-810.

27. Barzilai A, Goldberg I, Yulash M, et al. Silver-stained nucleolar organizer regions (AgNORs) as a prognostic value in malignant melanoma. Am J Dermatopathol 1998;20:473-7. 